\title{
Contributions of beliefs and processing fluency to the effect of relatedness on judgments of learning
}

\author{
Michael L. Mueller • Sarah K. Tauber • John Dunlosky
}

Published online: 27 November 2012

(C) Psychonomic Society, Inc. 2012

\begin{abstract}
Discovering how people judge their memories has been a major issue for metacognitive research for over 4 decades; many factors have been discovered that affect people's judgments, but exactly how those effects are mediated is poorly understood. For instance, the effect of word pair relatedness on judgments of learning (JOLs) has been repeatedly demonstrated, yet the underlying basis of this substantial effect is currently unknown. Thus, in three experiments, we assessed the contribution of beliefs and processing fluency. In Experiment 1, participants studied related and unrelated word pairs and made either prestudy JOLs or immediate JOLs. Participants gave higher estimates for related than for unrelated pairs, suggesting that participants' beliefs at least partially drive the relatedness effect on JOLs. Next, we evaluated the contribution of processing fluency to the relatedness effect either (1) by disrupting fluency by presenting half the pairs in an aLtErNaTiNg format (Experiment 2) or (2) by measuring how fluently participants processed pairs at study and statistically estimating the degree to which conceptual fluency mediated the effects of relatedness on JOLs (Experiment 3). Results from both experiments indicated that fluency contributes minimally to the relatedness effect. Taken together, these results indicate that people's beliefs about how relatedness influences memory are responsible for mediating the relationship between relatedness and JOLs. In general, empirically establishing what mediates the effects of other factors on people's judgments remains a major agenda for advancing theory of metacognitive monitoring.
\end{abstract}

M. L. Mueller · S. K. Tauber · J. Dunlosky $(\bowtie)$

Department of Psychology, Kent State University, Kent, OH 44242, USA

e-mail: jdunlosk@kent.edu
Keywords Beliefs · Fluency · Judgments of learning · Relatedness

Judgments of learning (JOLs) are people's predictions about the likelihood of remembering recently studied materials and are now among the most highly investigated of all metacognitive judgments (Dunlosky \& Metcalfe, 2009). People use their JOLs to guide their learning, such as when they are associating names with faces or learning foreign language translation equivalents. Given the importance of JOLs for effective regulation of learning associations, understanding how people monitor their learning has been a major focus of metacognitive research. Over 40 years of research has established that people do not base their JOLs on direct access to associative strength in memory but, instead, infer memory strength on the basis of available cues (Koriat, 1997). Although many cues have been identified that influence people's JOLs, a key theoretical issue concerns how people use any cue to construct JOLs.

One cue in particular - relatedness of words within a pairhas a major influence on people's JOLs that are made immediately after study. For example, Hertzog, Kidder, PowellMoman, and Dunlosky (2002) had participants study word pairs that were either related (pasture-cow) or unrelated (salt-mayor). Immediately after studying each pair, participants made a JOL. JOLs were substantially higher for related $(M=82 \%)$ than for unrelated $(M=57 \%)$ pairs. A review of the literature on relatedness and immediate JOLs is presented in Table 1. As is evident from inspection of this table, the relatedness effect on JOLs is robust and large, and hence, pair relatedness is a relatively ideal factor to empirically explore how cues influence JOLs, which is a major goal of the present research.

Two factors may contribute to the effects of many cues on JOLs, including the relatedness effect: beliefs and 
Table 1 Immediate judgments of learning for related and unrelated pairs

\begin{tabular}{lll}
\hline Study & Related Unrelated \\
\hline
\end{tabular}

Castel, McCabe, \& Roediger (2007) ${ }^{1}$

Experiment 1-strongly related, unrelated

Experiment 2-strongly related, unrelated

72

72

27

Connor, Dunlosky, \& Hertzog (1997)

Younger adults

Older adults

Dunlosky \& Matvey $(2001)^{2}$

Experiment 1

Related/unrelated order

Unrelated/related order

Experiment 2

Related/unrelated order

Unrelated/related order

Experiment 3

Hertzog et al. (2002)

Experiment 1

Younger adults

Older adults

Experiment 2

Younger adults

Older adults

Hertzog, Sinclair, \& Dunlosky (2010)

Jang \& Nelson (2005) ${ }^{1}$

Experiment 1B

Presented once

Presented twice

Experiment 1D

Studied $8 \mathrm{~s}$

Studied $2 \mathrm{~s}$

Experiment 2

Presented once

Presented twice

Koriat \& Bjork (2005) ${ }^{1}$

Experiment 1-strongly related, unrelated

Experiment 2-forward related, unrelated

Experiment 3-a priori related, unrelated

Koriat \& Bjork (2006) $)^{1,3}$

Experiment 1-forward related, unrelated

Experiment 2-forward related, unrelated

Experiment 3-forward related, unrelated

Koriat, Bjork, Sheffer, \& Bar (2004) ${ }^{1}$

Koriat, Ma'ayan, Sheffer, \& Bjork (2006) $)^{1,3}$

Experiment 1-a priori related, unrelated

Experiment 2-forward related, unrelated

Experiment 3-forward related, unrelated

Koriat \& Nussinson (2009)

Rabinowitz, Ackerman, Craik, \& Hinchley (1982)

Younger adults
Table 1 (continued)

\begin{tabular}{lll}
\hline Study & Related & Unrelated \\
\hline Older adults & 72 & 45 \\
Rhodes \& Castel (2008) & & \\
$\quad$ Large font size & 77 & 29 \\
$\quad$ Small font size & 72 & 25 \\
Soderstrom \& McCabe (2011) & & \\
$\quad$ Low value & & \\
$\quad$ Before & 54 & 26 \\
$\quad$ After & 55 & 31 \\
$\quad$ High value & & \\
$\quad$ Before & 67 & 40 \\
$\quad$ After & 69 & 46 \\
Tiede \& Leboe (2009) & & \\
Experimenter-paced study & 65 & 30 \\
Self-paced study & 66 & 32 \\
\hline
\end{tabular}

Values are means for each pair type

${ }^{1}$ Values were estimated from a figure

${ }^{2}$ Participants studied either all of the related pairs or all of the unrelated pairs first

${ }^{3}$ Experiments used multiple study-test cycles; only values from the first cycle are reported

${ }^{4}$ Point values were given either before or after the pair was presented for study. Low-value items were worth 1 or 2 points, and high-value items were worth 5 or 6 points when correctly recalled

processing fluency. Concerning the former, people may have a belief that they will remember related pairs better than unrelated ones. This belief may be activated from longterm memory when a JOL is made, or it may be developed when people are asked to make JOLs for related and unrelated pairs. With respect to processing fluency, Kornell, Rhodes, Castel, and Tauber (2011) noted that "ease of processing is a fundamental heuristic in metacognition that guides, and biases, judgments about memory" (pp. 792 793). In the present case, perhaps the ease with which information is processed directly influences people's JOLs. For example, in contrast to reading two unrelated words, reading the first word in a related pair may facilitate reading the second word, and such conceptual fluency, in turn, could lead to higher JOLs for related than unrelated pairs.

Beliefs and processing fluency may contribute to the substantial relatedness effect, and both have been offered as post hoc explanations. Although Koriat, Bjork, Sheffer, and Bar (2004) proposed that both beliefs and fluency can impact JOLs, they concluded that immediate "JOLs are based predominantly - perhaps exclusively - on the subjective experience associated with processing fluency" (p. 653). Concerning beliefs, Dunlosky and Matvey (2001) speculated that "it seems reasonable that college students have learned that relatedness influences memory, so 
related items receive high JOLs" (p. 1190). Most recently, Soderstrom and McCabe (2011) noted that "the mechanism responsible for relatedness effects on JOLs is debated, as these could arise from fluency (i.e., differences in how easily the items are processed) or knowledge (i.e., an explicit theory about how relatedness affects memory)" (p. 1236).

To provide an initial evaluation of people's beliefs about relatedness and memory, we administered a survey to 23 college students; the survey described a hypothetical experiment in which students studied both related and unrelated pairs (adapted from Koriat et al., 2004). After reading about this experiment, participants estimated how many related and unrelated pairs would be recalled. ${ }^{1}$ Participants estimated that recall was higher for related $(M=68 \%, S E=4.0)$ than for unrelated $(M=36 \%, S E=3.0)$ pairs, $t(22)=9.27$, $p<.001$. These survey data indicate that people have a belief about the relative memorability of related and unrelated pairs. Nevertheless, people may not apply this belief when making pair-by-pair JOLs. For instance, most people - and even children-believe that we forget across time (Kreutzer, Leonard, \& Flavell, 1975; Rawson, Dunlosky, \& McDonald, 2002; Tauber \& Rhodes, 2012), yet adults do not consistently incorporate this belief into JOLs that are made for individual pairs (Koriat et al., 2004). Likewise, although college students believe that relatedness enhances memory, this belief may not be responsible for the relatedness effect on JOLs. The goal of Experiment 1 was to examine whether participants' beliefs about relatedness influence their JOLs, and in Experiments 2 and 3, we evaluated the degree to which processing fluency mediates the relatedness effect.

\section{Experiment 1}

In Experiment 1, we used Castel's (2008) prestudy JOLs, which were prompted with the pair type. In particular, prior to studying each pair, participants were shown the type of pair (related or unrelated) that would be presented next for study and were asked to judge the likelihood of recalling it. The pair was then presented for study. Prestudy JOLs cannot be affected by processing fluency; thus, if participants apply a belief about relatedness when making judgments, prestudy JOLs will be higher for related than unrelated pairs. By contrast, if processing fluency entirely drives the relatedness effect, immediate JOLs (which could be affected by fluency) will demonstrate the relatedness effect, but prestudy JOLs will not.

\footnotetext{
${ }^{1}$ For further details about this experiment, please contact the corresponding author.
}

Method

We used a 2 (pair type: related or unrelated) $\times 2$ (judgment type: immediate or prestudy JOL) mixed-model factorial design, with pair type as the within-participant factor and judgment type as the between-participants factor. Forty-nine undergraduates participated to partially fulfill a course requirement and were randomly assigned to the immediate $(n=25)$ or prestudy $(n=24)$ JOL group.

Sixty paired associates from Rhodes and Castel (2008) were used. Half were related to one another, and half were unrelated. Participants studied each pair (presented in a random order) for $5 \mathrm{~s}$ and made a JOL for each pair on a scale from $0 \%$ to $100 \%$, indicating the likelihood that they would remember the second word when given the first. In the prestudy JOL group, participants made a JOL before studying each pair and were given the following prompt: "You are about to study a Related (Unrelated) word pair, please rate how likely you are to remember it." In the immediate JOL group, participants made a JOL (with the same prompt) after they had studied each pair. After all pairs had been studied and judged, participants then took a cued recall test.

\section{Results and discussion}

Mean percentage of correct recall is presented in Table 2. A 2 (pair type) $\times 2$ (judgment type) ANOVA revealed that recall was significantly greater for related than for unrelated pairs, $F(1,47)=484.06, M S E=7.67, p<.001, \eta_{\mathrm{p}}{ }^{2}=.91$. Recall was also greater after immediate than after prestudy JOLs, $F(1,47)=8.91, M S E=0.318, p=.004, \eta_{\mathrm{p}}^{2}=.16$; thus, making immediate JOLs had a minor reactive effect on recall, perhaps due to extra time processing pairs. The interaction was not significant.

As is shown in Fig. 1, relatedness had a substantial influence on JOLs, $F(1,47)=90.20, M S E=28,422.31$, $p<.001, \eta_{\mathrm{p}}{ }^{2}=.66$. The main effect of judgment type was not significant, $F=0.55$. The significant interaction, $F(1$, $47)=8.08, M S E=2,546.96, p=.007, \eta_{\mathrm{p}}{ }^{2}=.16$, indicated that the effect of relatedness was greater on immediate JOLs, $d=1.96$, than on prestudy JOLs, $d=1.01$. These outcomes suggest that people used a belief about relatedness in making their JOLs, because relatedness influenced prestudy JOLs. An alternative explanation is that people's beliefs about relatedness have no influence on JOLs but that, after experience studying some of the pairs, differential processing fluency leads them to eventually give higher JOLs (prestudy and immediate) to related pairs. To evaluate this possibility, we examined the first JOL made in the prestudy JOL group, which revealed that prior to studying any word pair, the mean prestudy JOL was $65(S E=5.1)$ for related pairs and $34(S E=5.0)$ for unrelated pairs, $t(22)$ 
Table 2 Mean percentage of correct recall

\begin{tabular}{lllllc}
\hline Judgment type & \multicolumn{2}{l}{ Standard format } & & \multicolumn{2}{l}{ Alternating format } \\
\cline { 2 - 3 } \cline { 5 - 6 } \cline { 5 - 6 } & Related & Unrelated & & Related & Unrelated \\
\hline Experiment 1 & & & & \\
$\quad$ Immediate JOL & $83.5(3.5)$ & $25.9(2.9)$ & & $x^{1}$ & $\mathrm{x}$ \\
$\quad \begin{array}{l}\text { Prestudy JOL } \\
\text { Experiment 2 }\end{array}$ & $70.4(3.6)$ & $16.1(2.9)$ & & $\mathrm{x}$ & $\mathrm{x}$ \\
$\quad$ Immediate JOL & $82.4(1.9)$ & $30.0(2.7)$ & $79.0(2.4)$ & $23.2(2.3)$ \\
Experiment 3 & & & & $\mathrm{x}$ \\
$\quad$ Immediate JOL & $78.3(2.7)$ & $17.5(2.7)$ & $\mathrm{x}$ & $\mathrm{x}$ \\
\hline
\end{tabular}

Values are means for each pair type and judgment type. Standard errors of the mean are in parentheses

${ }^{1}$ Alternating format was not used in Experiment 1 or 3

$=4.06, p<.001, d=1.74$. This outcome further supports the hypothesis that people's beliefs about relatedness produce the relatedness effect on JOLs.

\section{Experiment 2}

In Experiment 1, the effect of relatedness was larger on immediate JOLs than on prestudy JOLs (Fig. 1), which may occur because part of the relatedness effect on standard JOLs is mediated by differential processing fluency. In Experiment 2, we examined the contribution of fluency by using a method developed by Rhodes and Castel (2008). In their studies, participants gave higher JOLs to words

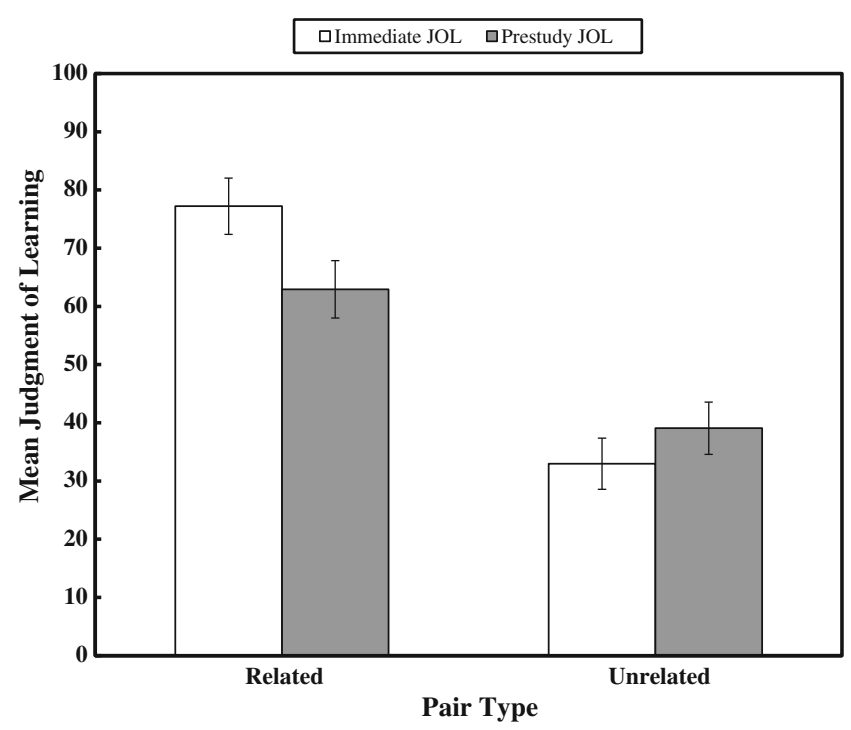

Fig. 1 Judgment-of-learning magnitude for each pair type and for each judgment type for Experiment 1. Error bars represent standard errors of the means presented in larger font than to those presented in smaller font. This effect was attributed to large words being processed more fluently, and to evaluate this hypothesis, they disrupted processing fluency by presenting words in an aLtErNaTiNg format. Consistent with their hypothesis, the font size effect disappeared when the words were presented in alternating format.

Using the same method, we investigated whether disrupting processing fluency would diminish the relatedness effect on JOLs. Given that we have found evidence that beliefs about relatedness influence JOLs, we expected a relatedness effect, regardless of the presentation format. However, if processing fluency also drives this effect on JOLs, disrupting processing fluency will decrease its magnitude.

\section{Method}

Fifty-two undergraduates were recruited to fulfill a partial course requirement. We used a 2 (pair type) $\times 2$ (presentation format: normal or alternating) within-participant design. The pairs from Experiment 1 were used. Fifteen pairs from each type were randomly assigned to be presented with an alternating format. We used two formats: One was an alternating format alone (e.g., BuZz-BeE), and the other was an alternating format with a gray-colored Haesttenschweiler font (Alter \& Oppenheimer, 2009). These formats had the same influence on JOLs and recall, so the data were combined and are referred to as alternating format. Participants studied the pairs, made immediate JOLs, and then took a cued recall test. Given that we did not use prestudy JOLs in Experiments 2 and 3, participants were not told which pair type they had just studied when they made JOLs, which is the standard prompt for JOLs.

Results and discussion

Mean percentage of correct recall is presented in Table 2. A $2 \times 2$ ANOVA revealed that recall was significantly greater for related than unrelated pairs, $F(1,51)=791, M S E=15.22, p<$ $.001, \eta_{\mathrm{p}}{ }^{2}=.94$, and was greater for pairs presented in normal format than for those presented in alternating format, $F(1,51)=11.73, M S E=0.137, p=.001, \eta_{\mathrm{p}}{ }^{2}=.19$.

As is shown in Fig. 2, JOLs were higher for related than for unrelated pairs, $F(1,51)=173.07, M S E=67,949.95$, $p<.001, \eta_{\mathrm{p}}{ }^{2}=.77$, and were higher for pairs presented in normal format than for those presented in alternating format, $F(1,51)=8.6, M S E=365.17, p=.005, \eta_{\mathrm{p}}{ }^{2}=.14$. Most important, the interaction was not significant, suggesting 


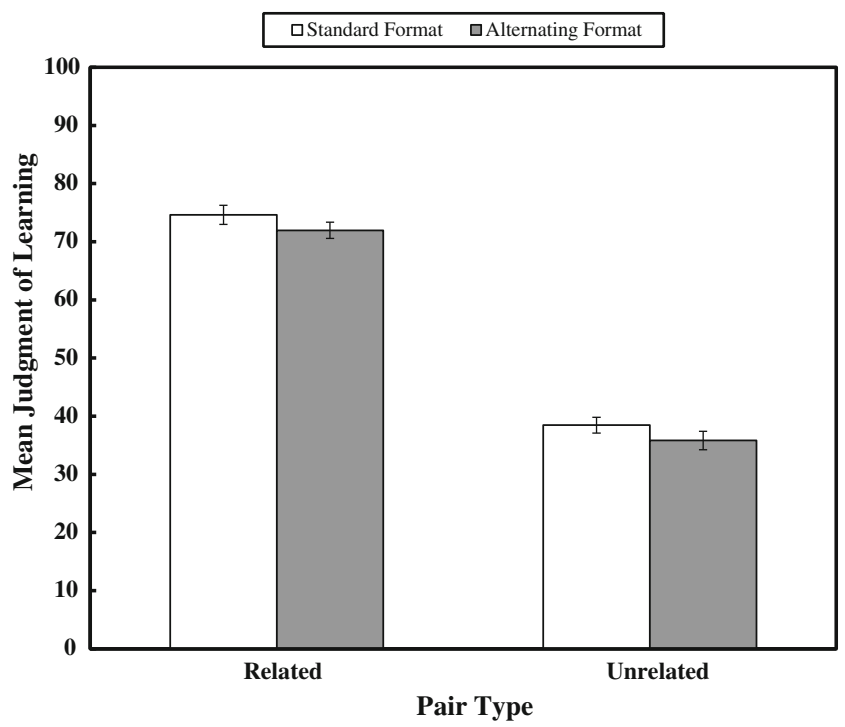

Fig. 2 Judgment-of-learning magnitude for each pair type and for each judgment type for Experiment 2. Error bars represent withinparticipant standard errors of the means (Loftus \& Masson, 1994)

that differences in processing fluency contribute minimally to the relatedness effect on JOLs.

\section{Experiment 3}

In Experiment 2, disrupting processing fluency by presenting words in aLtErNaTiNg format did not diminish the relatedness effect, although JOLs were lower for pairs presented in alternating format than for those presented in normal format. Thus, alternating format does influence JOLs, but processing fluency apparently does not contribute to the relatedness effect. One possibility is that another kind of fluency contributes (Alter \& Oppenheimer, 2009). For instance, alternating format presumably would undermine perceptual-processing fluency, yet conceptual fluencywith the meaning of the cue priming the related targetmay not be disrupted by alternating format and, hence, could still contribute to the relatedness effect. To measure conceptual-processing fluency, we had participants complete a lexical decision task using related, unrelated, and nonword pairs. In particular, the cue (always a word) and target of a pair were presented sequentially (some targets were nonwords), and participants were to quickly decide whether the target was a word or a nonword. After making the lexical decision, a JOL was made. As was expected, lexical decision response times were shorter for related than for unrelated pairs. Accordingly, using a correlational analysis, we evaluated the degree to which differences in conceptual fluency (as measured by response times) mediated the relationship between relatedness and JOLs.
Method

\section{Participants and materials}

Thirty-five undergraduates participated to fulfill a partial course requirement. Five participants were excluded because their performance on the lexical decision task was below $80 \%$. Items from Experiment 1, which were used to demonstrate the relatedness effects under scrutiny, were again used; namely, a subset of 20 related and 20 unrelated pairs were chosen, and the remaining word pairs were used to construct 20 word-nonword pairs (e.g., lime-arage).

\section{Procedure}

Participants completed a lexical decision task in which they decided whether the second word in each pair was a word or a nonword. The items were presented in a random order for each participant, with the constraint that no more than three items from any type (related, unrelated, nonword) appeared consecutively. Before each trial, participants were instructed to get ready by moving their fingers to the keys corresponding to a word ("Z") or a nonword ("M"), and then a fixation point appeared for $1 \mathrm{~s}$ to indicate where the cue would appear. The fixation point was replaced by the cue word, which remained on the screen for $1 \mathrm{~s}$. A second fixation point then appeared $(1 \mathrm{~s})$ to indicate where the target would appear. After the target appeared, participants were instructed to press " $Z$ " if it was a word or an " $M$ " if it was a nonword. Participants were instructed to make this decision as quickly as possible without making errors. Both words remained on the screen until participants had made their decision. Next, they were asked to make a JOL for that word pair. After completing this process for all pairs, participants took a cued recall test for the related and unrelated pairs.

Results and discussion

Recall was greater for related than unrelated pairs (Table 2), $t(29)=16.8, p<.001, \eta_{\mathrm{p}}{ }^{2}=.94$. JOLs differed by word type, $F$ $(2,58)=165.36, M S E=30,359.38, p<.001, \eta_{\mathrm{p}}{ }^{2}=.85$ : related $(M=82.58, S E=2.43)>$ unrelated $(M=49.40, S E=3.64)>$ nonword $(M=18.98, S E=3.54)$ pairs, all $t \mathrm{~s}>9.44, p \mathrm{~s}<.001$. Lexical decision response times also differed by word type, as was expected, $F(2,58)=9.64, M S E=0.464, p<.001$, $\eta_{\mathrm{p}}{ }^{2}=.25$ : related $(M=1.00 \mathrm{~s}, S E=0.08)<$ unrelated $(M=$ $1.11 \mathrm{~s}, S E=0.09)<$ nonword $(M=1.25 \mathrm{~s}, S E=0.14)$ pairs, all $t \mathrm{~s}>2.08, p \mathrm{~s}<.05$.

To evaluate the hypothesis that fluency mediates the relatedness effect on JOLs, we computed correlations between each participant's JOLs and relatedness, JOLs and lexical decision response times, and JOLs and relatedness, while 
controlling for lexical decision response times. Nonword data were not relevant to evaluating this hypothesis, so they were excluded. Consistent with the main effects described above, the mean correlations (Pearson $r$ ) were .66 between JOLs and relatedness, $t(27)=17.17, p<.001$, and -.21 between JOLs and response times, $t(27)=5.5, p<.001$. Most important, after controlling for lexical decision response time, the correlation between JOLs and relatedness was .65, $t(27)=17.32$, $p<.001$, which did not differ from the zero-order correlation between relatedness and JOLs. Thus, although conceptual processing fluency was related to JOLs, the differences in fluency did not mediate the relatedness effect.

\section{General discussion}

The present experiments investigated the degree to which beliefs and processing fluency contribute to the relatedness effect on JOLs. To evaluate their contribution empirically, we adapted methodologies used and developed by other researchers (Castel, 2008; Koriat et al., 2004; Rhodes \& Castel, 2008). In a survey study, participants estimated higher recall for related than for unrelated pairs, and in Experiment 1, prestudy JOLs made only with information about the relatedness of the pair revealed a relatedness effect, albeit slightly decreased in magnitude, as compared with immediate JOLs. This evidence supports the hypothesis that beliefs contribute substantially to the relatedness effect on JOLs. In Experiment 2, disrupting processing fluency by presenting words in aLtErNaTiNg format did not diminish the relatedness effect, although JOLs were lower for pairs presented in alternating format than for those presented in normal format. Thus, alternating format does influence JOLs, but perceptual processing apparently does not contribute to the relatedness effect. In Experiment 3, we examined the contribution of conceptual-processing fluency (as measured by lexical decision times), and as was expected, processing was more fluent for related than unrelated pairs. More important, such differences in processing fluency did not account for any of the relatedness effect on JOLs.

As was noted by Soderstrom and McCabe (2011), there is a debate about whether beliefs or fluency produces the relatedness effect on JOLs, and as was discussed above, our research provides empirical evidence relevant to estimating their contribution toward resolving the debate. We encourage such debates with regard to the many other cues that influence JOLs, as well as other metacognitive judgments. The challenge here will be to use empirical methods to explore the joint contribution of beliefs and fluency, which, in some cases, contribute in nonintuitive ways. For instance, a negative relationship occurs between retrieval latency and JOLs (e.g., Benjamin, Bjork, \& Schwartz,
1998; Serra \& Dunlosky, 2005), and some have speculated that this relationship occurs because the subjective experience of fluent retrieval has a nonconscious and direct effect on JOLs. However, when this speculation has been empirically evaluated, it has not fared well. Namely, evidence indicates that retrieval latency affects JOLs via people's belief that responses that take longer to retrieve (vs. are quickly retrieved) will be less likely recalled when later tested (Matvey, Dunlosky, \& Guttentag, 2001). So, in contrast to intuition, subjective fluency does not need to mediate latency-JOL relationships, which can result from beliefs about the relationship between latency and recall.

In conclusion, despite the number of cues that have been shown to influence JOLs and other metacognitive judgments (Dunlosky \& Metcalfe, 2009), few studies have included an evaluation of why these effects occur. Moreover, the degree to which fluency or beliefs mediate the effects of a given cue on JOLs may change across different contexts and conditions, yet such possibilities have largely been unexplored. Fortunately, given the number of methods available to more directly investigate the joint contribution of fluency and beliefs on JOLs, debates about their relative contributions can be resolved toward developing a general theory of beliefs, fluency, and metacognitive judgments that is empirically grounded. Toward this end, the present results demonstrate that people's beliefs largely-if not entirely-mediate the substantial effect of pair relatedness on JOLs.

Author Note We would like to thank Melissa Bishop, Andrea Courie, Nina Tubin, Sarah Turner, and Sri Siddhi Upadhyay for their assistance with data collection. Thanks to Matthew Rhodes for sharing ideas (and stimuli) that led to the design of Experiment 3.

This research was supported by the James S. McDonnell Foundation 21st Century Science Initiative in Bridging Brain, Mind, and Behavior Collaborative Award.

\section{References}

Alter, A. L., \& Oppenheimer, D. M. (2009). Uniting the tribes of fluency to form a metacognitive nation. Personality and Social Psychology Review, 13, 219-235. doi:10.1177/ 1088868310367640

Benjamin, A. S., Bjork, R. A., \& Schwartz, B. L. (1998). The mismeasure of memory: When retrieval fluency is misleading as a metamnemonic index. Journal of Experimental Psychology. General, 127, 55-68. doi:10.1037/0096-3445.127.1.55

Castel, A. D. (2008). Metacognition and learning about primacy and recency effects in free recall: The utilization of intrinsic and extrinsic cues when making judgments of learning. Memory and Cognition, 36, 429-437. doi:10.3758/MC.36.2.429

Castel, A. D., McCabe, D. P., \& Roediger, H. L., III. (2007). Illusions of competence and overestimation of associative memory for identical items: Evidence from judgments of learning. 
Psychonomic Bulletin and Review, 14, 107-111. doi:10.1177/ 1088868309341564

Connor, L. T., Dunlosky, J., \& Hertzog, C. (1997). Age-related differences in absolute but not relative metamemory accuracy. Psychology and Aging, 12, 50-71. doi:10.1037/0882-7974.12.1.50

Dunlosky, J., \& Matvey, G. (2001). Empirical analysis of the intrinsicextrinsic distinction of judgments of learning (JOLs): Effects of relatedness and serial position on JOLs. Journal of Experimental Psychology: Learning, Memory, and Cognition, 27, 1180-1191. doi:10.1037/0278-7393.27.5.1180

Dunlosky, J., \& Metcalfe, J. (2009). Metacognition. Thousand Oaks: Sage Publications, Inc.

Hertzog, C., Kidder, D. P., Powell-Moman, A., \& Dunlosky, J. (2002). Aging and monitoring associative learning: Is monitoring accuracy spared or impaired? Psychology and Aging, 17, 209-225. doi:10.1037/0882-7974.17.2.209

Hertzog, C., Sinclair, S. M., \& Dunlosky, J. (2010). Age differences in the monitoring of learning: Cross-sectional evidence of spared resolution across the adult life span. Developmental Psychology, 46, 939-948. doi:10.1037/a0019812

Jang, Y., \& Nelson, T. O. (2005). How many dimensions underlie judgments of learning and recall? Evidence from state-trace methodology. Journal of Experimental Psychology. General, 134, 308-326. doi:10.1037/0096-3445.134.3.308

Koriat, A. (1997). Monitoring one's own knowledge during study: A cue-utilization approach to judgments of learning. Journal of Experimental Psychology. General, 126, 349-370. doi:10.1037/ 0096-3445.126.4.349

Koriat, A., \& Bjork, R. A. (2005). Illusions of competence in monitoring one's knowledge during study. Journal of Experimental Psychology: Learning, Memory, and Cognition, 31, 187-194. doi:10.1037/0278-7393.31.2.187

Koriat, A., \& Bjork, R. A. (2006). Illusions of competence during study can be remedied by manipulations that enhance learners' sensitivity to retrieval conditions at test. Memory and Cognition, 34, 959-972. doi:10.3758/BF03193244

Koriat, A., Bjork, R. A., Sheffer, L., \& Bar, S. K. (2004). Predicting one's own forgetting: The role of experience-based and theorybased processes. Journal of Experimental Psychology. General, 133, 643-656. doi:10.1037/0096-3445.133.4.643

Koriat, A., Ma'ayan, H., Sheffer, L., \& Bjork, R. A. (2006). Exploring a mnemonic debiasing account of the underconfidence-with-practice effect. Journal of Experimental Psychology: Learning, Memory, and Cognition, 32, 595-608. doi:10.1037/0278-7393.32.3.595

Koriat, A., \& Nussinson, R. (2009). Attributing study effort to datadriven and goal-driven effects: Implications for metacognitive judgments. Journal of Experimental Psychology: Learning, Memory, and Cognition, 35, 1338-1343. doi:10.1037/a0016374

Kornell, N., Rhodes, M. G., Castel, A. D., \& Tauber, S. K. (2011). The ease-of-processing heuristic and stability bias: Dissociating memory, memory beliefs, and memory judgment. Psychological Science, 22, 787-794. doi:10.1177/0956797611407929

Kreutzer, M. A., Leonard, C., \& Flavell, J. H. (1975). An interview study of children's knowledge about memory. Monographs of the Society for Research in Child Development, 40, 1-60. doi:10.2307/1165955

Loftus, G. R., \& Masson, M. E. J. (1994). Using confidence intervals in within-subject designs. Psychonomic Bulletin and Review, 1, 476-490.

Matvey, G., Dunlosky, J., \& Guttentag, R. (2001). Fluency of retrieval at study affects judgments of learning (JOLs): An analytic or nonanalytic basis for JOLs? Memory and Cognition, 29, 222233. doi:10.3758/BF03194916

Rabinowitz, J. C., Ackerman, B. P., Craik, F. I. M., \& Hinchley, J. L. (1982). Aging and metamemory: The roles of relatedness and imagery. Journal of Gerontology, 37, 688-695. doi:10.1093/ geronj/37.6.688

Rawson, K. A., Dunlosky, J., \& McDonald, S. L. (2002). Influences of metamemory on performance predictions for text. The Quarterly Journal of Experimental Psychology, 55A, 505-524. doi:10.1080/ 02724980143000352

Rhodes, M. G., \& Castel, A. D. (2008). Memory predictions are influenced by perceptual information: Evidence for metacognitive illusions. Journal of Experimental Psychology. General, 137(4), 615-625. doi:10.1037/a0013684

Serra, M. J., \& Dunlosky, J. (2005). Does retrieval fluency contribute to the underconfidence-with-practice effect? Journal of Experimental Psychology: Learning, Memory, and Cognition, 31, 12581266. doi:10.1037/0278-7393.31.6.1258

Soderstrom, N. C., \& McCabe, D. P. (2011). The interplay between value and relatedness as bases for metacognitive monitoring and control: Evidence for agenda-based monitoring. Journal of Experimental Psychology: Learning, Memory, and Cognition, 37(5), 1236-1242. doi:10.1037/a0023548

Tauber, S. K., \& Rhodes, M. G. (2012). Measuring memory monitoring with judgements of retention (JORs). Quarterly Journal of Experimental Psychology. doi:10.1080/17470218.2012.656665

Tiede, H. L., \& Leboe, J. P. (2009). Illusions of competence for phonetically, orthographically, and semantically similar word pairs. Canadian Journal of Experimental Psychology/Revue Canadienne de Psychologie Expérimentale, 63(4), 294-302. doi:10.1037/a0015717 